University of Tennessee Law

Legal Scholarship Repository: A Service of the Joel A. Katz Library

UTK Law Faculty Publications

7-2017

2017 Transactional Clinic Conference - June 1-2, 2017

Philadelphia. Results of a Survey of Conference Participants on

Skills, Competencies, Learning Outcomes and Assessments (And

Other Transactional Clinic Demographics)

Brian Krumm

Follow this and additional works at: https://ir.law.utk.edu/utklaw_facpubs

Part of the Law Commons 
2017 Transactional Clinic Conference - June 1-2, 2017, Philadelphia Results of a Survey of Conference Participants on Skills, Competencies, Learning Outcomes and Assessments (and Other Transactional Clinic Demographics)

\author{
Barbara Wagner \\ Northern Kentucky University Chase College of Law \\ Gerard O'Connor \\ Boston University - School of Law
}

Christyne J. Vachon

University of Massachusetts School of Law at Dartmouth

Brian Kingley Krumm

University of Tennessee College of Law 


\section{Transactional Clinic Conference - June 1-2, 2017, Philadelphia \\ Results of a Survey of Conference Participants on \\ Skills, Competencies, Learning Outcomes and Assessments \\ (and Other Transactional Clinic Demographics)}

A total of 31 respondents completed the survey. Results are provided below. Percentages have been rounded, and may not total $100 \%$. Some minor grammatical clean-up in comments, where possible.

\section{Please choose the best description of how you teach and assess the following competencies, based on your view on which are most important in evaluating a transactional clinic student's performance.}

\begin{tabular}{|c|c|c|c|c|c|c|c|}
\hline & $\begin{array}{l}\text { I teach and } \\
\text { assess this: very } \\
\text { important in } \\
\text { evaluation/grade }\end{array}$ & $\begin{array}{l}\text { I teach and } \\
\text { assess this: } \\
\text { somewhat } \\
\text { important in } \\
\text { evaluation/grade }\end{array}$ & $\begin{array}{l}\text { I teach and } \\
\text { assess this: but } \\
\text { not significant } \\
\text { in evaluation/ } \\
\text { grade }\end{array}$ & $\begin{array}{l}\text { I teach this } \\
\text { but don't } \\
\text { assess it }\end{array}$ & $\begin{array}{l}\text { I assess this } \\
\text { but don't } \\
\text { teach it: not } \\
\text { significant in } \\
\text { evaluation/ } \\
\text { grade }\end{array}$ & $\begin{array}{l}\text { I assess this } \\
\text { but don't } \\
\text { teach it: } \\
\text { significant in } \\
\text { evaluation/ } \\
\text { grade }\end{array}$ & Total \\
\hline $\begin{array}{l}\text { Professional Responsibility and } \\
\text { Relationships (Awareness, } \\
\text { conscientiousness, concern and } \\
\text { sense of responsibility regarding } \\
\text { professional choices and } \\
\text { consequences; understanding the } \\
\text { lawyer's role in advising a new } \\
\text { enterprise) }\end{array}$ & $\begin{array}{l}39 \% \\
12\end{array}$ & $\begin{array}{c}42 \% \\
13\end{array}$ & $\begin{array}{c}6 \% \\
2\end{array}$ & $\begin{array}{c}13 \% \\
4\end{array}$ & $\begin{array}{c}0 \% \\
0\end{array}$ & $\begin{array}{c}0 \% \\
0\end{array}$ & 31 \\
\hline $\begin{array}{l}\text { Clinical Judgment (Ability to } \\
\text { identify and diagnose legal issues } \\
\text { relevant to client representation) }\end{array}$ & $\begin{array}{l}57 \% \\
17\end{array}$ & $\begin{array}{c}37 \% \\
11\end{array}$ & $\begin{array}{c}3 \% \\
1\end{array}$ & $\begin{array}{c}0 \% \\
0\end{array}$ & $\begin{array}{c}0 \% \\
0\end{array}$ & $\begin{array}{c}3 \% \\
1\end{array}$ & 30 \\
\hline $\begin{array}{l}\text { Legal Reasoning (Ability to } \\
\text { analyze a legal problem and } \\
\text { synthesize law and facts, apply } \\
\text { legal concepts to client-specific } \\
\text { facts) }\end{array}$ & $\begin{array}{l}61 \% \\
19\end{array}$ & $\begin{array}{l}35 \% \\
11\end{array}$ & $\begin{array}{c}0 \% \\
0\end{array}$ & $\begin{array}{c}0 \% \\
0\end{array}$ & $\begin{array}{c}0 \% \\
0\end{array}$ & $\begin{array}{c}3 \% \\
1\end{array}$ & 31 \\
\hline $\begin{array}{l}\text { Theoretical Perspective (Ability } \\
\text { to see legal issues in a broader } \\
\text { social and political context) }\end{array}$ & $\begin{array}{c}7 \% \\
2\end{array}$ & $\begin{array}{c}22 \% \\
6\end{array}$ & $\begin{array}{c}15 \% \\
4\end{array}$ & $\begin{array}{c}41 \% \\
11\end{array}$ & $\begin{array}{c}11 \% \\
3\end{array}$ & $\begin{array}{c}4 \% \\
1\end{array}$ & 27 \\
\hline $\begin{array}{l}\text { Communication (Ability to listen } \\
\text { to clients, understand their legal } \\
\text { issues and respond both orally } \\
\text { and in writing, with accuracy, } \\
\text { clarity and economy; ability to } \\
\text { convey complex legal concepts in } \\
\text { plain English) }\end{array}$ & $\begin{array}{c}87 \% \\
27\end{array}$ & $\begin{array}{c}10 \% \\
3\end{array}$ & $\begin{array}{c}0 \% \\
0\end{array}$ & $\begin{array}{c}3 \% \\
1\end{array}$ & $\begin{array}{c}0 \% \\
0\end{array}$ & $\begin{array}{c}0 \% \\
0\end{array}$ & 31 \\
\hline $\begin{array}{l}\text { Management of Effort (Ability to } \\
\text { use time, resources and energy } \\
\text { with awareness of competing } \\
\text { priorities in a way that produces } \\
\text { optimal legal services; ability to } \\
\text { handle variety of matters, meet } \\
\text { deadlines and commitments; } \\
\text { adjust to changing priorities) }\end{array}$ & $\begin{array}{c}55 \% \\
17\end{array}$ & $\begin{array}{c}29 \% \\
9\end{array}$ & $\begin{array}{c}3 \% \\
1\end{array}$ & $\begin{array}{c}0 \% \\
0\end{array}$ & $\begin{array}{c}6 \% \\
2\end{array}$ & $\begin{array}{c}6 \% \\
2\end{array}$ & 31 \\
\hline $\begin{array}{l}\text { Risk Identification and } \\
\text { Assessment (Ability to identify } \\
\text { legal risks for clients, understand } \\
\text { business risks and appreciate } \\
\text { client's risk tolerance, including } \\
\text { appreciation of law practice } \\
\text { economics, e.g., billable hour cost } \\
\text { and level of certainty) }\end{array}$ & $\begin{array}{c}20 \% \\
6\end{array}$ & $\begin{array}{c}47 \% \\
14\end{array}$ & $\begin{array}{c}23 \% \\
7\end{array}$ & $\begin{array}{c}7 \% \\
2\end{array}$ & $\begin{array}{c}3 \% \\
1\end{array}$ & $\begin{array}{c}0 \% \\
0\end{array}$ & 30 \\
\hline
\end{tabular}




\begin{tabular}{|c|c|c|c|c|c|c|c|}
\hline & $\begin{array}{l}\text { I teach and } \\
\text { assess this: very } \\
\text { important in } \\
\text { evaluation/grade }\end{array}$ & $\begin{array}{l}\text { I teach and } \\
\text { assess this: } \\
\text { somewhat } \\
\text { important in } \\
\text { evaluation/grade }\end{array}$ & $\begin{array}{l}\text { I teach and } \\
\text { assess this: but } \\
\text { not significant } \\
\text { in evaluation/ } \\
\text { grade }\end{array}$ & $\begin{array}{l}\text { I teach this } \\
\text { but don't } \\
\text { assess it }\end{array}$ & $\begin{array}{l}\text { I assess this } \\
\text { but don't } \\
\text { teach it: not } \\
\text { significant in } \\
\text { evaluation/ } \\
\text { grade }\end{array}$ & $\begin{array}{l}\text { I assess this } \\
\text { but don't } \\
\text { teach it: } \\
\text { significant in } \\
\text { evaluation/ } \\
\text { grade }\end{array}$ & Total \\
\hline $\begin{array}{l}\text { Judgment and Common Sense } \\
\text { (Ability to develop scope of work, } \\
\text { level of refinement based on } \\
\text { client's needs, including when to } \\
\text { stop or continue research, when } \\
\text { to seek guidance) }\end{array}$ & $\begin{array}{c}39 \% \\
12\end{array}$ & $\begin{array}{c}32 \% \\
10\end{array}$ & $\begin{array}{c}6 \% \\
2\end{array}$ & $\begin{array}{c}6 \% \\
2\end{array}$ & $\begin{array}{c}10 \% \\
3\end{array}$ & $\begin{array}{c}6 \% \\
2\end{array}$ & 31 \\
\hline $\begin{array}{l}\text { Thinking Outside the Box (Ability } \\
\text { to expand efforts beyond the } \\
\text { bounds of the client assignment } \\
\text { itself and providing additional } \\
\text { value to the client) }\end{array}$ & $\begin{array}{c}13 \% \\
4\end{array}$ & $\begin{array}{c}32 \% \\
10\end{array}$ & $\begin{array}{c}19 \% \\
6\end{array}$ & $\begin{array}{c}16 \% \\
5\end{array}$ & $\begin{array}{c}19 \% \\
6\end{array}$ & $\begin{array}{c}0 \% \\
0\end{array}$ & 31 \\
\hline $\begin{array}{l}\text { Cultural Awareness and Social } \\
\text { Justice (Sensitivity to different } \\
\text { cultural norms, including cultural, } \\
\text { socio-economic status, access to } \\
\text { legal resources and ability to act } \\
\text { appropriately with clients, staff, } \\
\text { faculty, other students and } \\
\text { others) }\end{array}$ & $\begin{array}{c}7 \% \\
2\end{array}$ & $\begin{array}{c}25 \% \\
7\end{array}$ & $\begin{array}{c}25 \% \\
7\end{array}$ & $\begin{array}{c}32 \% \\
9\end{array}$ & $\begin{array}{c}4 \% \\
1\end{array}$ & $\begin{array}{c}7 \% \\
2\end{array}$ & 28 \\
\hline $\begin{array}{l}\text { Classroom Discussion and } \\
\text { Rounds (Questions and } \\
\text { comments on assigned materials } \\
\text { and clinic assignments, makes } \\
\text { relevant contributions that } \\
\text { promote deeper analysis) }\end{array}$ & $\begin{array}{c}33 \% \\
10\end{array}$ & $\begin{array}{c}43 \% \\
13\end{array}$ & $\begin{array}{c}13 \% \\
4\end{array}$ & $\begin{array}{c}0 \% \\
0\end{array}$ & $\begin{array}{c}7 \% \\
2\end{array}$ & $\begin{array}{c}3 \% \\
1\end{array}$ & 30 \\
\hline
\end{tabular}

\section{Others/Comments:}

The ability to work as a team and bring out the best in their teammate. It Client satisfaction with effort. Effectiveness at delivering presentations, Preparation, reliability/dependability.

Initiative in case mgmt, not simply acting like an associate expecting to be assigned tasks

Growth is the most important category in terms of our assessment of students. Have they improved from beginning to end regardless of starting level.

Thinking strategically and tactically, which really seems to be included in a combo of questions above.

\section{Ability to work in a team}

I also consider identification of interdisciplinary issues. 


\section{Please choose the best description of how you teach and assess the following skills, based on your view on which are most important in evaluating a transactional clinic student's performance.}

\begin{tabular}{|c|c|c|c|c|c|c|c|}
\hline & $\begin{array}{l}\text { I teach and } \\
\text { assess this: very } \\
\text { important in } \\
\text { evaluation/ } \\
\text { grade }\end{array}$ & $\begin{array}{l}\text { I teach and } \\
\text { assess this: } \\
\text { somewhat } \\
\text { important in } \\
\text { evaluation/grade }\end{array}$ & $\begin{array}{l}\text { I teach and } \\
\text { assess this: but } \\
\text { not significant } \\
\text { in evaluation/ } \\
\text { grade }\end{array}$ & $\begin{array}{l}\text { I teach this } \\
\text { but don't } \\
\text { really } \\
\text { assess it }\end{array}$ & $\begin{array}{l}\text { I assess this } \\
\text { but don't } \\
\text { really teach } \\
\text { it: not } \\
\text { significant in } \\
\text { evaluation/ } \\
\text { grade }\end{array}$ & $\begin{array}{l}\text { I assess this } \\
\text { but don't } \\
\text { teach it: } \\
\text { significant in } \\
\text { evaluation/ } \\
\text { grade }\end{array}$ & Total \\
\hline $\begin{array}{l}\text { Client Interviewing and Counseling } \\
\text { (Planning, active listening, } \\
\text { establishing rapport with the client, } \\
\text { identifying issues, effectiveness in } \\
\text { executing clients' choice) }\end{array}$ & $\begin{array}{c}81 \% \\
25\end{array}$ & $\begin{array}{c}16 \% \\
5\end{array}$ & $\begin{array}{c}3 \% \\
1\end{array}$ & $\begin{array}{c}0 \% \\
0\end{array}$ & $\begin{array}{c}0 \% \\
0\end{array}$ & $\begin{array}{c}0 \% \\
0\end{array}$ & 31 \\
\hline $\begin{array}{l}\text { Research and Information Gathering } \\
\text { (Ability to obtain accurate } \\
\text { information about client's operations } \\
\text { and goals, thorough factual legal } \\
\text { research, and an awareness of } \\
\text { client's deadlines and time } \\
\text { constraints) }\end{array}$ & $\begin{array}{c}42 \% \\
13\end{array}$ & $\begin{array}{c}52 \% \\
16\end{array}$ & $\begin{array}{c}3 \% \\
1\end{array}$ & $\begin{array}{c}0 \% \\
0\end{array}$ & $\begin{array}{c}0 \% \\
0\end{array}$ & $\begin{array}{c}3 \% \\
1\end{array}$ & 31 \\
\hline $\begin{array}{l}\text { Problem Solving and Negotiation } \\
\text { (Ability to identify and comprehend } \\
\text { legal issues and risks based on } \\
\text { client's goals and objectives; ability } \\
\text { to effectively counsel clients and } \\
\text { persuasively communicate client's } \\
\text { position to third parties, incl. } \\
\text { negotiate) }\end{array}$ & $\begin{array}{c}53 \% \\
16\end{array}$ & $\begin{array}{c}33 \% \\
10\end{array}$ & $\begin{array}{c}3 \% \\
1\end{array}$ & $\begin{array}{c}10 \% \\
3\end{array}$ & $\begin{array}{c}0 \% \\
0\end{array}$ & $\begin{array}{c}0 \% \\
0\end{array}$ & 30 \\
\hline $\begin{array}{l}\text { Communication and Drafting (Ability } \\
\text { to write and draft clearly, } \\
\text { grammatically, without typos, well- } \\
\text { organized, in a variety of contexts, } \\
\text { including emails, memos, contracts, } \\
\text { engagement letters) }\end{array}$ & $\begin{array}{c}87 \% \\
27\end{array}$ & $\begin{array}{c}10 \% \\
3\end{array}$ & $\begin{array}{c}0 \% \\
0\end{array}$ & $\begin{array}{c}0 \% \\
0\end{array}$ & $\begin{array}{c}0 \% \\
0\end{array}$ & $\begin{array}{c}3 \% \\
1\end{array}$ & 31 \\
\hline $\begin{array}{l}\text { Supervision and Project } \\
\text { Management (Ability to manage } \\
\text { client assignments effectively, } \\
\text { update clients on status, prioritize } \\
\text { projects; aware of need for input } \\
\text { from supervisor and build in time for } \\
\text { supervisor review) }\end{array}$ & $\begin{array}{c}52 \% \\
16\end{array}$ & $\begin{array}{c}35 \% \\
11\end{array}$ & $\begin{array}{c}10 \% \\
3\end{array}$ & $\begin{array}{c}3 \% \\
1\end{array}$ & $\begin{array}{c}0 \% \\
0\end{array}$ & $\begin{array}{c}0 \% \\
0\end{array}$ & 31 \\
\hline $\begin{array}{l}\text { Work Product (Ability to plan and } \\
\text { counsel client on final work product } \\
\text { to be delivered; prepare timely, clear } \\
\text { document - organized, grammatical, } \\
\text { no typos, well-formatted; ability to } \\
\text { create work product from scratch } \\
\text { and use forms) }\end{array}$ & $\begin{array}{c}77 \% \\
24\end{array}$ & $\begin{array}{c}19 \% \\
6\end{array}$ & $\begin{array}{c}0 \% \\
0\end{array}$ & $\begin{array}{c}0 \% \\
0\end{array}$ & $\begin{array}{c}0 \% \\
0\end{array}$ & $\begin{array}{c}3 \% \\
1\end{array}$ & 31 \\
\hline $\begin{array}{l}\text { Office Administration and File } \\
\text { Maintenance (Adherence to office } \\
\text { procedures, keep appointments, } \\
\text { retain and organize all documents } \\
\text { and notes in file systems. Timely } \\
\text { record billable hours.) }\end{array}$ & $\begin{array}{c}23 \% \\
7\end{array}$ & $\begin{array}{c}39 \% \\
12\end{array}$ & $\begin{array}{c}23 \% \\
7\end{array}$ & $\begin{array}{c}6 \% \\
2\end{array}$ & $\begin{array}{c}10 \% \\
3\end{array}$ & $\begin{array}{c}0 \% \\
0\end{array}$ & 31 \\
\hline $\begin{array}{l}\text { General Professionalism (Attention } \\
\text { to professionalism when working } \\
\text { with: clients, support staff, internal } \\
\text { and external attorneys, and the } \\
\text { media and public; understand the } \\
\text { role of a lawyer) }\end{array}$ & $\begin{array}{c}48 \% \\
15\end{array}$ & $\begin{array}{c}39 \% \\
12\end{array}$ & $\begin{array}{c}10 \% \\
3\end{array}$ & $\begin{array}{c}0 \% \\
0\end{array}$ & $\begin{array}{c}0 \% \\
0\end{array}$ & $\begin{array}{c}3 \% \\
1\end{array}$ & 31 \\
\hline
\end{tabular}




\begin{tabular}{|c|c|c|c|c|c|c|c|}
\hline & $\begin{array}{l}\text { I teach and } \\
\text { assess this: very } \\
\text { important in } \\
\text { evaluation/ } \\
\text { grade }\end{array}$ & $\begin{array}{l}\text { I teach and } \\
\text { assess this: } \\
\text { somewhat } \\
\text { important in } \\
\text { evaluation/grade }\end{array}$ & $\begin{array}{l}\text { I teach and } \\
\text { assess this: but } \\
\text { not significant } \\
\text { in evaluation/ } \\
\text { grade }\end{array}$ & $\begin{array}{l}\text { I teach this } \\
\text { but don't } \\
\text { really } \\
\text { assess it }\end{array}$ & $\begin{array}{l}\text { I assess this } \\
\text { but don't } \\
\text { really teach } \\
\text { it: not } \\
\text { significant in } \\
\text { evaluation/ } \\
\text { grade }\end{array}$ & $\begin{array}{l}\text { I assess this } \\
\text { but don't } \\
\text { teach it: } \\
\text { significant in } \\
\text { evaluation/ } \\
\text { grade }\end{array}$ & Total \\
\hline $\begin{array}{l}\text { Ethical Issues (Sensitivity to potential } \\
\text { ethical issues in representing } \\
\text { clients: role of counselor, drafter, } \\
\text { advocate; client confidentiality, } \\
\text { competence, scope of } \\
\text { representation, conflict of interest, } \\
\text { beginning and end of engagement) }\end{array}$ & $\begin{array}{c}42 \% \\
13\end{array}$ & $\begin{array}{c}35 \% \\
11\end{array}$ & $\begin{array}{c}10 \% \\
3\end{array}$ & $\begin{array}{c}10 \% \\
3\end{array}$ & $\begin{array}{c}0 \% \\
0\end{array}$ & $\begin{array}{c}3 \% \\
1\end{array}$ & 31 \\
\hline $\begin{array}{l}\text { Computer Skills and Other Business } \\
\text { Basics (facility with word, including } \\
\text { formatting, track changes, comparing } \\
\text { documents, metadata, and other } \\
\text { skills like excel, formatting a business } \\
\text { letter, business jargon) }\end{array}$ & $\begin{array}{c}3 \% \\
1\end{array}$ & $\begin{array}{c}24 \% \\
7\end{array}$ & $\begin{array}{c}24 \% \\
7\end{array}$ & $\begin{array}{c}21 \% \\
6\end{array}$ & $\begin{array}{c}28 \% \\
8\end{array}$ & $\begin{array}{c}0 \% \\
0\end{array}$ & 29 \\
\hline $\begin{array}{l}\text { Reflecting on Performance (Ability to } \\
\text { reflect upon and identify one's own } \\
\text { strengths and weaknesses; accept } \\
\text { constructive criticism; and modify } \\
\text { performance based upon critique or } \\
\text { self assessment) }\end{array}$ & $\begin{array}{c}23 \% \\
7\end{array}$ & $\begin{array}{c}33 \% \\
10\end{array}$ & $\begin{array}{c}20 \% \\
6\end{array}$ & $\begin{array}{c}10 \% \\
3\end{array}$ & $\begin{array}{c}7 \% \\
2\end{array}$ & $\begin{array}{c}7 \% \\
2\end{array}$ & 30 \\
\hline $\begin{array}{l}\text { Class Attendance and Participation } \\
\text { (Effective class performance and } \\
\text { ability to work with other student } \\
\text { attorneys to effectively serve client; } \\
\text { timely for meetings with clients and } \\
\text { supervisor). }\end{array}$ & $\begin{array}{c}48 \% \\
15\end{array}$ & $\begin{array}{c}29 \% \\
9\end{array}$ & $\begin{array}{c}13 \% \\
4\end{array}$ & $\begin{array}{c}0 \% \\
0\end{array}$ & $\begin{array}{c}3 \% \\
1\end{array}$ & $\begin{array}{c}6 \% \\
2\end{array}$ & 31 \\
\hline
\end{tabular}

\section{[no comments]}

\section{Do you have any other comments on skills, competencies, learning outcomes or assessment in your clinic?}

I use Q Qextensive Qualtrics surveys ad mid-semester and end of semester to get their own perspectives on their performance of the grading criteria. I also solicit feedback on the lessons they are learning and ways that they would improve the program.

Important to note that we grade Credit /no credit for the clinical work. We can't accept anything less than A-level work. But we do grade the classroom component, because we want to incentivize class participation and attendance.

The greatest lesson we want to students to walk away with is the ability to teach themselves -- to learn how to learn. This is what they'll have to do in practice so we structure the experience so that they must do this in the Clinic.

As I filled this out, it occurred to me that some of the skills and competencies measure into evaluation only if they are "bad." Example: you don't get extra credit for being on time, but you can definitely lose points for being late. The other issue is some students never run into an ethical (or some other) issues, while others may have them in spades. Hard to evaluate something that not all students deal with.

Our clinic does not assess "skills" and "competencies" separately. It assesses "learning outcomes," which encompass both skills and competencies.

We do a Final Portfolio, which includes reflecting on three pieces of work product: (1) best, (2) worst, and (3) a creative value add. 
4. What types of clients does your clinic represent - or other types of projects does the clinic undertake? (choose all that apply).

\begin{tabular}{|c|c|}
\hline Answer Choices & Responses \\
\hline $\begin{array}{l}\text { would-be entrepreneurs who want to set up a for-profit or social enterprise } \\
\text { business }\end{array}$ & $\begin{array}{c}97 \% \\
30\end{array}$ \\
\hline small businesses (first year or two).- less than $\$ 100,000$ annual revenue & $\begin{array}{c}94 \% \\
29\end{array}$ \\
\hline small businesses (more than two years old) - less than $\$ 100,000$ annual revenue & $\begin{array}{c}71 \% \\
22\end{array}$ \\
\hline larger businesses - $\$ 100,000$ annual revenue or more & $\begin{array}{c}19 \% \\
6\end{array}$ \\
\hline founders for nonprofit formation & $\begin{array}{c}68 \% \\
21\end{array}$ \\
\hline existing nonprofits & $\begin{array}{c}68 \% \\
21\end{array}$ \\
\hline government agencies & $\begin{array}{c}10 \% \\
3\end{array}$ \\
\hline primarily students from my university & $\begin{array}{c}3 \% \\
1\end{array}$ \\
\hline primarily students, faculty and staff from my or another university & $\begin{array}{c}23 \% \\
7\end{array}$ \\
\hline primarily clients from a specific neighborhood or region & $\begin{array}{c}42 \% \\
13\end{array}$ \\
\hline $\begin{array}{l}\text { Other (please specify), including types of projects beyond direct client } \\
\text { representation: } \\
\text { client referrals from various entrepreneurial resources } \\
\text { In addition to full-service representation, UCB Practicum provides community } \\
\text { workshops on the law, regular office hours, in the summer we will launch an } \\
\text { online legal resource library for entrepreneurs. The services are focused on } \\
\text { entrepreneurs in California's East Bay and Central Valley } \\
\text { Definitely represent students from my university but its not the primary } \\
\text { client. Also target certain neighborhoods and corridors, but again, not } \\
\text { primary. } \\
\text { Clients include students from my university, other universities and } \\
\text { neighborhood based clients } \\
\text { student entrepreneurs } \\
\text { Artists, inventors not interested in setting up companies, and I do represent } \\
\text { student entrepreneurs and the university technology transfer office, although } \\
\text { neither "primarily" }\end{array}$ & $\begin{array}{c}26 \% \\
8\end{array}$ \\
\hline
\end{tabular}


5. What types of projects does your clinic undertake for clients or other constituencies?

\begin{tabular}{|c|c|c|c|c|c|}
\hline & $\begin{array}{l}\text { All students } \\
\text { do this at } \\
\text { least once } \\
\end{array}$ & $\begin{array}{l}\text { Most students } \\
\text { do this at least } \\
\text { once }\end{array}$ & 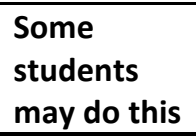 & $\begin{array}{l}\text { Students do } \\
\text { this rarely or } \\
\text { never }\end{array}$ & Total \\
\hline Interview client & $\begin{array}{c}97 \% \\
30\end{array}$ & $\begin{array}{c}0 \% \\
0\end{array}$ & $\begin{array}{c}3 \% \\
1\end{array}$ & $\begin{array}{c}0 \% \\
0\end{array}$ & 31 \\
\hline Draft intake memo to file & $\begin{array}{c}68 \% \\
21\end{array}$ & $\begin{array}{c}10 \% \\
3\end{array}$ & $\begin{array}{c}19 \% \\
6\end{array}$ & $\begin{array}{c}3 \% \\
1\end{array}$ & 31 \\
\hline Draft or mark up engagement letter & $\begin{array}{l}77 \% \\
24\end{array}$ & $\begin{array}{c}19 \% \\
6\end{array}$ & $\begin{array}{c}0 \% \\
0\end{array}$ & $\begin{array}{c}3 \% \\
1\end{array}$ & 31 \\
\hline Draft a contract & $\begin{array}{c}35 \% \\
11\end{array}$ & $\begin{array}{c}55 \% \\
17\end{array}$ & $\begin{array}{c}10 \% \\
3\end{array}$ & $\begin{array}{c}0 \% \\
0\end{array}$ & 31 \\
\hline Review, revise or comment on a contract & $\begin{array}{c}29 \% \\
9\end{array}$ & $\begin{array}{c}58 \% \\
18\end{array}$ & $\begin{array}{c}13 \% \\
4\end{array}$ & $\begin{array}{c}0 \% \\
0\end{array}$ & 31 \\
\hline $\begin{array}{l}\text { Write a memo to a client to describe an } \\
\text { area of law or answer a question }\end{array}$ & $\begin{array}{c}45 \% \\
14\end{array}$ & $\begin{array}{c}39 \% \\
12\end{array}$ & $\begin{array}{c}16 \% \\
5\end{array}$ & $\begin{array}{c}0 \% \\
0\end{array}$ & 31 \\
\hline Form a legal entity & $\begin{array}{c}29 \% \\
9\end{array}$ & $\begin{array}{c}32 \% \\
10\end{array}$ & $\begin{array}{c}32 \% \\
10\end{array}$ & $\begin{array}{c}6 \% \\
2\end{array}$ & 31 \\
\hline $\begin{array}{l}\text { Other corporate filing (dissolution, amend } \\
\text { articles, etc.) }\end{array}$ & $\begin{array}{c}10 \% \\
3\end{array}$ & $\begin{array}{c}23 \% \\
7\end{array}$ & $\begin{array}{c}61 \% \\
19\end{array}$ & $\begin{array}{c}6 \% \\
2\end{array}$ & 31 \\
\hline Prepare or advise on Form 1023 or $1023 \mathrm{EZ}$ & $\begin{array}{c}3 \% \\
1\end{array}$ & $\begin{array}{c}16 \% \\
5\end{array}$ & $\begin{array}{c}48 \% \\
15\end{array}$ & $\begin{array}{c}32 \% \\
10\end{array}$ & 31 \\
\hline Other nonprofit tax advice & $\begin{array}{c}6 \% \\
2\end{array}$ & $\begin{array}{c}19 \% \\
6\end{array}$ & $\begin{array}{c}32 \% \\
10\end{array}$ & $\begin{array}{c}42 \% \\
13\end{array}$ & 31 \\
\hline Copyright advice or filing & $\begin{array}{c}0 \% \\
0\end{array}$ & $\begin{array}{c}20 \% \\
6\end{array}$ & $\begin{array}{c}60 \% \\
18\end{array}$ & $\begin{array}{c}20 \% \\
6\end{array}$ & 30 \\
\hline Trademark advice or filing & $\begin{array}{c}10 \% \\
3\end{array}$ & $\begin{array}{c}35 \% \\
11\end{array}$ & $\begin{array}{c}39 \% \\
12\end{array}$ & $\begin{array}{c}16 \% \\
5\end{array}$ & 31 \\
\hline Patent advice or filing & $\begin{array}{c}0 \% \\
0\end{array}$ & $\begin{array}{c}10 \% \\
3\end{array}$ & $\begin{array}{c}13 \% \\
4\end{array}$ & $\begin{array}{c}77 \% \\
23\end{array}$ & 30 \\
\hline Tax advice (NOT nonprofit) & $\begin{array}{c}0 \% \\
0\end{array}$ & $\begin{array}{c}7 \% \\
2\end{array}$ & $\begin{array}{c}21 \% \\
6\end{array}$ & $\begin{array}{c}72 \% \\
21\end{array}$ & 29 \\
\hline Negotiate & $\begin{array}{c}6 \% \\
2\end{array}$ & $\begin{array}{c}16 \% \\
5\end{array}$ & $\begin{array}{c}65 \% \\
20\end{array}$ & $\begin{array}{c}13 \% \\
4\end{array}$ & 31 \\
\hline Carry out a business transaction & $\begin{array}{c}13 \% \\
4\end{array}$ & $\begin{array}{c}32 \% \\
10\end{array}$ & $\begin{array}{c}35 \% \\
11\end{array}$ & $\begin{array}{c}19 \% \\
6\end{array}$ & 31 \\
\hline $\begin{array}{l}\text { Present educational programs on legal } \\
\text { matters in the community }\end{array}$ & $\begin{array}{c}42 \% \\
13\end{array}$ & $\begin{array}{c}6 \% \\
2\end{array}$ & $\begin{array}{c}35 \% \\
11\end{array}$ & $\begin{array}{c}16 \% \\
5\end{array}$ & 31 \\
\hline
\end{tabular}

All students contribute content to our online legal resource library -- every team is expected to do one workshop in the community on a legal issue, for example, how to protect intellectual property, and we record it, and load it into the legal resource library.

Most students will counsel a client about potential solutions to problems. 
6. How many students participate in the clinic at one time, per supervising faculty member?

\begin{tabular}{|c|c|}
\hline Answer Choices & Responses \\
\hline $5-7$ & $\begin{array}{c}17 \% \\
5\end{array}$ \\
\hline $7-9$ & $\begin{array}{c}33 \% \\
10\end{array}$ \\
\hline 9 or more & $\begin{array}{c}3 \% \\
1\end{array}$ \\
\hline always 8 & $\begin{array}{c}0 \% \\
0\end{array}$ \\
\hline variable: 4-10 & $\begin{array}{c}17 \% \\
5\end{array}$ \\
\hline $\begin{array}{l}\text { Other (please specify) } \\
\text { I teach two clinics, an } 8 \text {-student, } 6 \text { credit clinic and an } 8 \text {-student, } 3 \text { credit practicum } \\
\text { (with an outside IP co-counsel). } \\
\text { Usually } 8 \text {, but due to student interest ( } 54 \text { applicants [ } 1 / 4 \text { of eligible students] - all } \\
\text { qualified), so I added a full section of --8 during summer session and am temporarily } \\
\text { increasing the fall and spring to } 10 \text { each semester } \\
\text { We have two attorneys, including myself, and we have } 18 \text { students per term. Some of } \\
\text { those twins are returning students. Supervising attorney covers all of the full- } \\
\text { service representation client work, while the deputy attorney supervises all office } \\
\text { hour student participation and the content creation for the online legal resource } \\
\text { library. } \\
4 \text { students, one adjunct faculty member (half-time) } \\
\text { Full time faculty supervises } 8 / \text { semester; Adjunct supervises } 4 / \text { semester } \\
4 \\
\text { Ratio is } 8: 1 \text {; however, we will sometimes take on "advanced" clinic students, who only } \\
\text { do client work, which boosts the ratio somewhat. } \\
12 \\
3\end{array}$ & $\begin{array}{c}30 \% \\
9\end{array}$ \\
\hline Total & 30 \\
\hline
\end{tabular}

7. Approximately how many total students participated in your clinic in the most recent academic year?

\begin{tabular}{|c|c|}
\hline Answer Choices & Responses \\
\hline 10 or fewer & $7 \%$ \\
\hline \multirow[b]{3}{*}{$11-15$} & \\
\hline & $17 \%$ \\
\hline & 5 \\
\hline \multirow{2}{*}{$16-20$} & $33 \%$ \\
\hline & 10 \\
\hline \multirow{2}{*}{$21-30$} & $27 \%$ \\
\hline & 8 \\
\hline \multirow{2}{*}{$31-40$} & $10 \%$ \\
\hline & 3 \\
\hline \multirow{2}{*}{ more than 40} & $7 \%$ \\
\hline & 2 \\
\hline Total & 30 \\
\hline
\end{tabular}


8. Do you grade the classroom component of your clinic separately from the clinic/client component?

\begin{tabular}{|c|c|}
\hline Answer Choices & Responses \\
\hline \multirow{2}{*}{ yes } & $32 \%$ \\
& 10 \\
\hline \multirow{2}{*}{ no } & $68 \%$ \\
& 21 \\
\hline Total & 31 \\
\hline
\end{tabular}

9. How many credit hours does a student typically receive for participating in the clinic?

\begin{tabular}{|l|c|}
\hline Answer Choices & Responses \\
\hline 2 or 3 & $13 \%$ \\
& 4 \\
\hline 4 or 5 & $27 \%$ \\
\hline 6 or 7 & $83 \%$ \\
\hline 8 or more & 13 \\
\hline $\begin{array}{l}\text { totally variable (anywhere from } 1 \text { to } 10 \text { or } \\
\text { more) }\end{array}$ & $7 \%$ \\
\hline $\begin{array}{c}\text { Other (please specify): } \\
6 \text { or } 3 \\
\text { Two for the seminar, and four for the } \\
\text { clinical work }\end{array}$ & 0 \\
\hline 4 for clinic, additional 2 for required class & $10 \%$ \\
\hline Total & 30 \\
\hline
\end{tabular}

10. How many hours per credit hour are students expected to spend on clinic work?

\begin{tabular}{|c|c|}
\hline Answer Choices & Responses \\
\hline \multirow{2}{*}{42.5} & $27 \%$ \\
\hline & 8 \\
\hline \multirow{2}{*}{$43-49$} & $27 \%$ \\
\hline & 8 \\
\hline \multirow{2}{*}{ at least 50} & $30 \%$ \\
\hline & 9 \\
\hline \multicolumn{2}{|l|}{ Other (please specify) } \\
\hline \multirow{3}{*}{$\begin{array}{l}\text { Four hours per week per credit, so I total of } 16 \text { hours per week on the } \\
\text { client work } \\
60 \text { (law school requirement) } \\
10 \text { hrs per week }\end{array}$} & $17 \%$ \\
\hline & \\
\hline & \\
\hline
\end{tabular}


The question doesn't make sense for the way we calculate hours. It's 3

hours per credit hour $\times 14$ credit hours (for 2 semesters) $=42$

hours, but that's a minimum.

Not certain. 40 ?

Total

30

11. What is the approximate size of a $1 \mathrm{~L}$ class at your law school?

\begin{tabular}{|c|c|}
\hline Answer Choices & Responses \\
\hline less than 100 & $\begin{array}{c}6 \% \\
2\end{array}$ \\
\hline 100-199 & $\begin{array}{c}39 \% \\
12\end{array}$ \\
\hline $200-299$ & $\begin{array}{c}32 \% \\
10\end{array}$ \\
\hline $300-399$ & $\begin{array}{c}13 \% \\
4\end{array}$ \\
\hline 400 or more & $\begin{array}{c}10 \% \\
3\end{array}$ \\
\hline Total & 31 \\
\hline
\end{tabular}

12. Does your law school have an evening program?

\begin{tabular}{|l|c|}
\hline Answer Choices & Responses \\
\hline no & $74 \%$ \\
& 23 \\
\hline \multirow{2}{*}{ yes } & $26 \%$ \\
& 8 \\
\hline Total & 31 \\
\hline
\end{tabular}

13. If your school has an evening program, are evening students eligible to participate in your clinic?

\begin{tabular}{|l|c|}
\hline Answer Choices & Responses \\
\hline \multirow{2}{*}{ yes, and they participate often } & $\begin{array}{c}12 \% \\
3\end{array}$ \\
\hline \multirow{2}{*}{ yes, and they participate occasionally } & $0 \%$ \\
& 0 \\
\hline \multirow{2}{*}{ yes, but they participate rarely } & $12 \%$ \\
& 3 \\
\hline \multirow{2}{*}{ no } & $0 \%$ \\
& 0 \\
\hline \multirow{2}{*}{ Not applicable (no evening program) } & $77 \%$ \\
& 20 \\
\hline Total & 26 \\
\hline
\end{tabular}

Class meets during the day, when they work; and our clients meet at night, when the students go to other classes.

So evening students rarely participate. 
14. How many transactional clinics does your law school offer?

\begin{tabular}{|c|c|}
\hline Answer Choices & Responses \\
\hline 1 & $\begin{array}{c}42 \% \\
13\end{array}$ \\
\hline 2 & $\begin{array}{c}35 \% \\
11\end{array}$ \\
\hline 3 & $\begin{array}{c}3 \% \\
1\end{array}$ \\
\hline 4 & $\begin{array}{c}6 \% \\
2\end{array}$ \\
\hline 5 or more & $\begin{array}{c}13 \% \\
4\end{array}$ \\
\hline Total & 31 \\
\hline
\end{tabular}

4 core clinics plus a few minor practicums and clinics

\section{Where is your clinic located? (check all that apply)}

\begin{tabular}{|l|c|}
\hline Answer Choices & Responses \\
\hline In the law school & $94 \%$ \\
29
\end{tabular}

satellite office in urban community

We have a location at the law school as well as in the metropolitan city we serve 
16. What prerequisites does your clinic have for students to participate?

\begin{tabular}{|c|c|c|c|c|c|}
\hline & $\begin{array}{l}\text { Required } \\
\text { (1 weighting) }\end{array}$ & $\begin{array}{l}\text { Recommended/ } \\
\text { preferred, but } \\
\text { can be waived } \\
\text { ( } 2 \text { weighting) }\end{array}$ & $\begin{array}{l}\text { Somewhat } \\
\text { desirable } \\
\text { ( } 3 \text { weighting) }\end{array}$ & Total & $\begin{array}{l}\text { Weighted } \\
\text { Average }\end{array}$ \\
\hline $\begin{array}{l}\text { Business Organizations (or } \\
\text { equivalent) }\end{array}$ & $\begin{array}{c}46 \% \\
11\end{array}$ & $\begin{array}{c}46 \% \\
11\end{array}$ & $\begin{array}{c}8 \% \\
2\end{array}$ & 24 & 1.63 \\
\hline Professional Responsibility & $\begin{array}{c}44 \% \\
8\end{array}$ & $\begin{array}{c}28 \% \\
5\end{array}$ & $\begin{array}{c}28 \% \\
5\end{array}$ & 18 & 1.83 \\
\hline Basic Tax & $\begin{array}{c}9 \% \\
1\end{array}$ & $\begin{array}{c}18 \% \\
2\end{array}$ & $\begin{array}{c}73 \% \\
8\end{array}$ & 11 & 2.64 \\
\hline Advanced/Corporate Tax & $\begin{array}{c}8 \% \\
1\end{array}$ & $\begin{array}{c}8 \% \\
1\end{array}$ & $\begin{array}{c}83 \% \\
10\end{array}$ & 12 & 2.75 \\
\hline Intellectual Property & $\begin{array}{c}11 \% \\
2\end{array}$ & $\begin{array}{c}32 \% \\
6\end{array}$ & $\begin{array}{c}58 \% \\
11\end{array}$ & 19 & 2.47 \\
\hline Contract Drafting & $\begin{array}{c}6 \% \\
1\end{array}$ & $\begin{array}{c}25 \% \\
4\end{array}$ & $\begin{array}{c}69 \% \\
11\end{array}$ & 16 & 2.63 \\
\hline $\begin{array}{l}\text { Transactional } \\
\text { Practice/Skills }\end{array}$ & $\begin{array}{c}15 \% \\
2\end{array}$ & $\begin{array}{c}31 \% \\
4\end{array}$ & $\begin{array}{c}54 \% \\
7\end{array}$ & 13 & 2.38 \\
\hline $\begin{array}{l}\text { Approx } 30 \text { credit hours } \\
\text { total law study (one full } \\
\text { year) }\end{array}$ & $\begin{array}{c}93 \% \\
13\end{array}$ & $\begin{array}{c}7 \% \\
1\end{array}$ & $\begin{array}{c}0 \% \\
0\end{array}$ & 14 & 1.07 \\
\hline $\begin{array}{l}\text { Approx } 60 \text { credit hours } \\
\text { total law study (two full } \\
\text { years) }\end{array}$ & $\begin{array}{c}62 \% \\
8\end{array}$ & $\begin{array}{c}23 \% \\
3\end{array}$ & $\begin{array}{c}15 \% \\
2\end{array}$ & 13 & 1.54 \\
\hline $\begin{array}{l}\text { Must be eligible for } \\
\text { student practice license }\end{array}$ & $\begin{array}{c}79 \% \\
11\end{array}$ & $\begin{array}{c}14 \% \\
2\end{array}$ & $\begin{array}{c}7 \% \\
1\end{array}$ & 14 & 1.29 \\
\hline $\begin{array}{l}\text { Must obtain student } \\
\text { practice license (if eligible) }\end{array}$ & $\begin{array}{c}82 \% \\
9\end{array}$ & $\begin{array}{c}0 \% \\
0\end{array}$ & $\begin{array}{c}18 \% \\
2\end{array}$ & 11 & 1.36 \\
\hline
\end{tabular}

no student practice rule in $\mathrm{MO}$ for transactional work

There is no student practice license or other prerequisites required, though next year we might require at least a business associations course

Negotiation; we only have 3Ls in the fall and at most half $2 \mathrm{Ls}$ in the Spring.

none

4th semester required

$2 \mathrm{~L}$ or $3 \mathrm{~L}$ status 
17. Approximately what percent of students at your law school who apply for your clinic are admitted to participate?

\begin{tabular}{|l|c|}
\hline Answer Choices & Responses \\
\hline All - and sometimes undersubscribed & $3 \%$ \\
& 1 \\
\hline $100 \%$ & $3 \%$ \\
& 1 \\
\hline $75 \%$ & 33. \\
& 10 \\
\hline $50 \%$ & $20 \%$ \\
\hline Less than 50\% & 6 \\
\hline Students are usually admitted for the semester/year they & $27 \%$ \\
apply & 8 \\
\hline Students are usually admitted, but not necessarily for the & $30 \%$ \\
semester/year they first apply & 9 \\
\hline Clinic assignments are based on allocation of all students & $10 \%$ \\
to various clinics - may not get first choice clinic & 3 \\
\hline Total Respondents: 30 & \\
\hline
\end{tabular}

I defer rising $2 \mathrm{Ls}$ and try to accommodate all qualified $1 \mathrm{Ls}$ [sic - presumably meant $3 \mathrm{Ls}$ ]

18. Are students permitted to participate in the clinic for a second time (beyond the standard - semester, quarter or year)?

\begin{tabular}{|l|c|}
\hline Answer Choices & Responses \\
\hline Never & $44 \%$ \\
& 11 \\
\hline On a space available basis & $24 \%$ \\
& 6 \\
\hline \multirow{2}{*}{ Often } & $20 \%$ \\
& 5 \\
\hline Always & $4 \%$ \\
& 1 \\
\hline Limited projects for pro bono & $8 \%$ \\
hours & 2 \\
\hline Total & 25 \\
\hline
\end{tabular}

Supervisor can invite students to continue

I wish

We offer an "Advanced Clinic" by invitation. It's fewer credits, 3-4, and graded.

I do take Independent Study students who have taken my clinic to continue to represent clients under my supervision

haven't dealt with this yet

A couple stay on for a second semester

Only upon invitation for credit ( $3 \mathrm{hrs}$ ) or pro bono hours Sometimes, for 2 credit hours 


\section{What sort of extra training do students receive before the beginning of the clinic?}

\begin{tabular}{|l|c|}
\hline Answer Choices & Responses \\
\hline None, all training is completed in classroom component & $43 \%$ \\
13 \\
\hline Orientation of up to 5 hours & $23 \%$ \\
& 7 \\
\hline \multirow{2}{*}{ Boot camp of 5 to 15 hours } & $27 \%$ \\
& 8 \\
\hline More than 15 hours & $7 \%$ \\
\hline Total & 2 \\
\hline
\end{tabular}

I have tried orientation of 5-6 hours but due to our Quarter system , it is very hard to schedule.

Intensive training day during first couple of weeks

Orientation for $21 / 2$ days during the first week of classes.

\section{Do students generally work with clients in pairs or individually?}

\begin{tabular}{|l|c|}
\hline Answer Choices & Responses \\
\hline $\begin{array}{l}\text { Students generally have their own client(s) or own project(s) for } \\
\text { client and work individually }\end{array}$ & $\begin{array}{c}40 \% \\
12\end{array}$ \\
\hline $\begin{array}{l}\text { Students generally work in pairs - same pair for all clients in } \\
\text { semester }\end{array}$ & $\begin{array}{c}50 \% \\
15\end{array}$ \\
\hline $\begin{array}{l}\text { Students generally work in pairs - but different pairings for } \\
\text { different clients }\end{array}$ & $3 \%$ \\
\hline Students generally work in groups of 3 or more & $7 \%$ \\
\hline Total & 30 \\
\hline
\end{tabular}

Students always work in teams of three

Students generally work on one client individually and one client in a pair

I will assign some complex matters to teams of two. I will also assign a client to two students and they will divide up assignments rather than work together.

Students have one individual and one paired client

Work individually with some clients and in pairs for others; occasionally in small groups 
21. Does your clinic operate over the summer?

\begin{tabular}{|l|c|}
\hline Answer Choices & Responses \\
\hline \multirow{2}{*}{ yes } & $32 \%$ \\
& 10 \\
\hline \multirow{2}{*}{ no } & $68 \%$ \\
& 21 \\
\hline Total & 31 \\
\hline
\end{tabular}

Operates without students.

This summer, for the first time, due to student demand Deputy attorney and supervising attorney cover all cases in the summer, and may except new cases if urgent need More limited capacity

For those clients who need it

\section{How do students in your clinic maintain files?}

\begin{tabular}{|l|c|}
\hline Answer Choices & Responses \\
\hline \multirow{2}{*}{ paper } & $0 \%$ \\
& 0 \\
\hline \multirow{2}{*}{ electronic } & $32 \%$ \\
& 10 \\
\hline \multirow{2}{*}{ both } & $68 \%$ \\
& 21 \\
\hline Total & 31 \\
\hline
\end{tabular}

Just switched to electronic-only

\section{Do your students communicate with clients via email?}

\begin{tabular}{|l|c|}
\hline Answer Choices & Responses \\
\hline No & $0 \%$ \\
& 0 \\
\hline Yes, through their individual (personal) email accounts & $6 \%$ \\
& 2 \\
\hline Yes, through school-issued email accounts to which they retain & $68 \%$ \\
access after their participation in the clinic ends & 21 \\
\hline Yes, through clinic-issued email accounts over which the clinic & $26 \%$ \\
maintains custody and control & 8 \\
\hline Total & 31 \\
\hline
\end{tabular}

School issued accounts continue for about one year after graduation

Our entire clinical program will adopt clinic-issued email accounts this coming year. 


\section{How do your students carry out legal work (research, drafting) for your clinic?}

\begin{tabular}{|l|c|}
\hline Answer Choices & Responses \\
\hline $\begin{array}{l}\text { Clinic-supplied computer with clinic-only drive - accessible only during clinic } \\
\text { participation }\end{array}$ & $10 \%$ \\
\hline $\begin{array}{l}\text { Cloud-based server (e.g., Cllo) with clinic-only access - accessible only during } \\
\text { clinic participation }\end{array}$ & $7 \%$ \\
\hline Both clinic-supplied and cloud-based options, as described above & 2 \\
\hline May use personal computer but required to delete all drafts and work & $5 \%$ \\
product after clinic participation (other than approved writing samples) & $40 \%$ \\
\hline May use personal computer but requested to delete all drafts and work & 12 \\
\hline product after clinic participation (other than approved writing samples) & $13 \%$ \\
\hline Personal computer with no limitations on deletion of work product & 4 \\
\hline Total & $13 \%$ \\
\hline
\end{tabular}

Students understand that client confidentiality responsibility continues after their participation in the clinic. Also cloud server for documents (NetDocuments) and timekeeping (Clio)

Also have access to clinic-supplied computer

ALSO use clinic and cloud based options

\section{What do you do when you encounter matters outside your clinic's capabilities?}

\begin{tabular}{|l|c|}
\hline Answer Choices & Responses \\
\hline Decline representation & $7 \%$ \\
& 2 \\
\hline Undertake partial representation (portion clinic can assist & $46 \%$ \\
with) & 13 \\
\hline Refer to private counsel or other resource recommended & $29 \%$ \\
by clinic & 8 \\
\hline Provide a list of several potential alternate private (or pro & $18 \%$ \\
bono) resources & 5 \\
\hline \multirow{2}{*}{ Refer to local bar association for recommendations } & $0 \%$ \\
\hline Total & 0 \\
\hline
\end{tabular}

We might to any or all of the above.

Whichever of the above seems appropriate under the circumstances

All of the above

If we can handle the majority of issues, we undertake partial representation. If that is inefficient for client, or we cannot handle the majority of issues, we will refer to programs and potential practitioners in the community.

We also accept representation but work with co-counsel with necessary expertise

Combination of the above answers would be more accurate

Depends--sometimes take portions if they exist, sometimes refer our entire matter or decline altogether 
I'd like to check more than one here. We may take the part we can, but we will refer to a list of other potential resources AND to the local bar

\section{What is the average grade awarded in your clinic?}

\begin{tabular}{|l|c|}
\hline Answer Choices & Responses \\
\hline Pass (based on pass fail scale) & $11 \%$ \\
& 3 \\
\hline Some form of A (or equivalent, & $63 \%$ \\
such as 3.5 and above) & 17 \\
\hline Some form of B (or equivalent, & $26 \%$ \\
such as 2.5 - 3.4 ) & 7 \\
\hline Some form of C (or equivalent, & $0 \%$ \\
such as 1.5 - 2.4) & 0 \\
\hline Total & 27 \\
\hline
\end{tabular}

Again, clinical work is based on credit versus no credit scale, seminar is based on class participation and attendance, so the seminar average is equivalent to $\mathrm{a} B+$

$\mathrm{B}+$

Pass and high pass, about $50 / 50$

Based on a 3.5 curve

A-

\section{What types of employment do your students typically pursue after graduation? (check all that apply)}

\begin{tabular}{|c|c|c|c|c|c|}
\hline & Often & Sometimes & Rarely & $\begin{array}{l}\text { Don't } \\
\text { know }\end{array}$ & Total \\
\hline Large Firm (over 100 attorneys) & $\begin{array}{c}50 \% \\
15\end{array}$ & $\begin{array}{c}23 \% \\
7\end{array}$ & $\begin{array}{c}17 \% \\
5\end{array}$ & $\begin{array}{c}10 \% \\
3\end{array}$ & 30 \\
\hline Medium Firm (20-99 attorneys) & $\begin{array}{c}21 \% \\
6\end{array}$ & $\begin{array}{c}71 \% \\
20\end{array}$ & $\begin{array}{c}0 \% \\
0\end{array}$ & $\begin{array}{c}7 \% \\
2\end{array}$ & 28 \\
\hline Small Firm (2-19 attorneys) & $\begin{array}{c}31 \% \\
9\end{array}$ & $\begin{array}{c}31 \% \\
9\end{array}$ & $\begin{array}{c}31 \% \\
9\end{array}$ & $\begin{array}{c}7 \% \\
2\end{array}$ & 29 \\
\hline Sole practitioner & $\begin{array}{c}7 \% \\
2\end{array}$ & $\begin{array}{c}26 \% \\
7\end{array}$ & $\begin{array}{c}56 \% \\
15\end{array}$ & $\begin{array}{c}11 \% \\
3\end{array}$ & 27 \\
\hline Court & $\begin{array}{c}3 \% \\
1\end{array}$ & $\begin{array}{c}41 \% \\
12\end{array}$ & $\begin{array}{c}45 \% \\
13\end{array}$ & $\begin{array}{c}10 \% \\
3\end{array}$ & 29 \\
\hline Government (non-court) & $\begin{array}{c}4 \% \\
1\end{array}$ & $\begin{array}{c}36 \% \\
10\end{array}$ & $\begin{array}{c}46 \% \\
13\end{array}$ & $\begin{array}{c}14 \% \\
4\end{array}$ & 28 \\
\hline JD Advantage & $\begin{array}{c}0 \% \\
0\end{array}$ & $\begin{array}{c}36 \% \\
10\end{array}$ & $\begin{array}{c}29 \% \\
8\end{array}$ & $\begin{array}{c}36 \% \\
10\end{array}$ & 28 \\
\hline $\begin{array}{l}\text { Continue in current job (for } \\
\text { evening students) }\end{array}$ & $\begin{array}{c}0 \% \\
0\end{array}$ & $\begin{array}{c}13 \% \\
2\end{array}$ & $\begin{array}{c}47 \% \\
7\end{array}$ & $\begin{array}{c}40 \% \\
6\end{array}$ & 15 \\
\hline Transactional law & $\begin{array}{c}64 \% \\
18\end{array}$ & $\begin{array}{c}25 \% \\
7\end{array}$ & $\begin{array}{c}4 \% \\
1\end{array}$ & $\begin{array}{c}7 \% \\
2\end{array}$ & 28 \\
\hline Non-transactional & $\begin{array}{c}4 \% \\
1\end{array}$ & $\begin{array}{c}73 \% \\
19\end{array}$ & $\begin{array}{c}15 \% \\
4\end{array}$ & $\begin{array}{c}8 \% \\
2\end{array}$ & 26 \\
\hline
\end{tabular}




\section{How long have you been engaged in teaching in a transactional clinic?}

\begin{tabular}{|l|c|}
\hline Answer Choices & Responses \\
\hline $\begin{array}{l}\text { Just finished my first } \\
\text { year }\end{array}$ & $\begin{array}{c}13 \% \\
4\end{array}$ \\
\hline 2-5 years & $45 \%$ \\
& 14 \\
\hline 6-10 years & $29 \%$ \\
& 9 \\
\hline more than 10 years & $13 \%$ \\
& 4 \\
\hline Total & 31 \\
\hline
\end{tabular}

19 years

29. Did you practice transactional law before your clinic teaching?

\begin{tabular}{|l|c|}
\hline Answer Choices & Responses \\
\hline No & $3 \%$ \\
& 1 \\
\hline 2 years or less & $6 \%$ \\
\hline 3-7 years & 2 \\
\hline 8-15 years & $32 \%$ \\
& 10 \\
\hline $16-30$ years & $23 \%$ \\
\hline more than 30 years & 7 \\
\hline Total & $26 \%$ \\
\hline
\end{tabular}

30. If we revised this survey and circulated it for broader input, are there any additional questions you would like to see added? Any you would like to delete or clarify?

For those of us teaching more than 1 clinical offering, would be helpful to clarify how to answer. Also, clarify the summer work question re: students/no students.

Contract structures, salary information, faculty standing related questions.

Whether clinics limit representation to clients who lack access to counsel.

Some questions should allow for multiple answers

I would like a copy of the entire form and answers.

The first two questions should have an option for neither teaching nor assessing a particular skill Include a question re whether the clinic lasts for one or two semesters; clarify credit hours question. How are clinics funded? Are they staffed with adjuncts or full time, non-tenure track instructors? 